\title{
Rayleigh instability of confined vortex droplets in critical superconductors
}

\author{
I. Lukyanchuk ${ }^{1 \star}$, V. M. Vinokur ${ }^{2}$, A. Rydh ${ }^{3}$, R. Xie ${ }^{2}$, M. V. Milošević ${ }^{4}$, U. Welp ${ }^{2}$, M. Zach ${ }^{2}$, Z. L. Xiao ${ }^{2}$, \\ G. W. Crabtree ${ }^{2}$, S. J. Bending ${ }^{5}$, F. M. Peeters ${ }^{4}$ and W. K. Kwok ${ }^{2}$
}

Depending on the Ginzburg-Landau parameter $\kappa_{r}$ superconductors can either be fully diamagnetic if $\kappa<1 / \sqrt{2}$ (type I superconductors) or allow magnetic flux to penetrate through Abrikosov vortices if $\kappa>1 / \sqrt{2}$ (type II superconductors; refs 1,2). At the Bogomolny critical point, $\kappa=\kappa_{\mathrm{c}}=1 / \sqrt{2}$, a state that is infinitely degenerate with respect to vortex spatial configurations arises ${ }^{3,4}$. Despite in-depth investigations of conventional type I and type II superconductors, a thorough understanding of the magnetic behaviour in the near-Bogomolny critical regime at $\kappa \sim \kappa_{\mathrm{c}}$ remains lacking. Here we report that in confined systems the critical regime expands over a finite interval of $\kappa$ forming a critical superconducting state. We show that in this state, in a sample with dimensions comparable to the vortex core size, vortices merge into a multi-quanta droplet, which undergoes Rayleigh instability ${ }^{5}$ on increasing $\kappa$ and decays by emitting single vortices. Superconducting vortices realize Nielsen-Olesen singular solutions of the Abelian Higgs model, which is pervasive in phenomena ranging from quantum electrodynamics to $\operatorname{cosmolog}^{6-9}$. Our study of the transient dynamics of Abrikosov-Nielsen-Olesen vortices in systems with boundaries promises access to non-trivial effects in quantum field theory by means of bench-top laboratory experiments.

The evolution of magnetic properties of an infinite superconductor when crossing $\kappa_{\mathrm{c}}$ is shown in Fig. 1. Type I superconductors with $\kappa<\kappa_{\mathrm{c}}$ expel magnetic field $H$ until it reaches a critical field $H_{\mathrm{c}}$ beyond which superconductivity is destroyed (Fig. 1b,e). In type II superconductors with $\kappa>\kappa_{\mathrm{c}}$, superconductivity extends into a wider region, $H_{\mathrm{c} 1}<H<H_{\mathrm{c} 2}$, where magnetic field penetrates the sample in the form of Abrikosov vortices, tiny filaments of the normal phase surrounded by encircling supercurrents (Fig. 1a,d), each carrying a quantum magnetic flux $\Phi_{0}=\pi \hbar / c e$.

Finite-size systems acquire new features enriching their phase diagram. Most importantly, type I superconductors fall into an intermediate state, comprising alternating domains of normal and superconducting phases with the period $d \simeq 10 \sqrt{D \xi}$ for $H \simeq 0.5 H_{\text {c }}$ (ref. 10), where $\xi$ is the coherence length and $D$ is the sample thickness. The intermediate state forms in the interval $(1-n) H_{c}<H<H_{c}(n<1$ is the shape-dependent demagnetization factor) triggered by the local magnetic field near the edges of the sample exceeding the critical value $H_{c}$ and locally destroying superconductivity (Fig. 1c,f). In type II superconductors, nucleation of superconductivity occurs first near the sample boundary at a specific surface critical field $H_{\mathrm{c} 3}>H_{\mathrm{c} 2}$. In type I superconductors $H_{\mathrm{c} 3}$ can exceed $H_{\mathrm{c}}$ if $\kappa \lesssim \kappa_{\mathrm{c}}$, as shown in Fig. 1b,e.
Near $\kappa$-induced criticality, with domains containing only a few flux quanta, the intermediate state is unstable towards breaking into an Abrikosov lattice and transient effects become important. To analyse transient behaviour, we consider a sample with $\kappa \lesssim \kappa_{\mathrm{c}}$ containing a single domain or droplet of the normal phase, that is, a sample with the lateral size $L$ comparable to the period $d$ of the domain structure. This droplet is nothing but a giant vortex with a normal core comprising several flux quanta ${ }^{11}$. Its critical fission occurs by splitting an $\mathrm{N}$-quanta droplet ( $\mathrm{Nq}$-droplet) into a $(N-1) q$-droplet and a single $1 q$ vortex moving away (see Fig. 2). To calculate the energy difference between the $N q$-droplet and the configuration consisting of the residual $(N-1) q$-droplet and the separated vortex, we construct a perturbation theory in the vicinity of the Bogomolny point over the small parameter $\gamma=\kappa^{2}-\kappa_{c}^{2}$ (Supplementary Information) and identify three contributions to the interaction energy (see Fig. 2c):

$$
U(l)=U_{\mathrm{e}}(l)+U_{\mathrm{i}}(l)+U_{\mathrm{c}}(l)
$$

where $l$ is the distance between the vortex and the droplet. The intrinsic interaction energy of the $(N-1) q$-droplet with the separated vortex calculated in ref. 12 (see also Supplementary Information and ref. 13) is

$$
U_{\mathrm{e}}(l)=-\gamma \frac{\alpha}{4 \sqrt{N}} \frac{D}{\lambda} H_{\mathrm{c}}^{2} \lambda l^{2}, \quad \alpha \simeq 0.3
$$

where $\lambda$ is the London electromagnetic screening length. This term yields attraction at $\gamma<0$ as expected. Magnetostatic repulsion energy due to stray fields generated by vortices near the sample surface is

$$
U_{\mathrm{i}}(l)=-2 \zeta(N) H_{\mathrm{c}}^{2} \lambda l^{2}, \quad \zeta(N) \simeq 0.04 \sqrt{N}
$$

Finally, the confinement energy due to interaction of the external field $H$ with the vortex, holding the droplet together is

$$
U_{c}(l)=2 \eta \frac{H}{H_{c}} \frac{\lambda}{L} H_{c}^{2} \lambda l^{2}, \quad \eta \simeq 0.52
$$

As follows from equation (4), decreasing the field reduces the confinement strengths. At some threshold field, the repulsive forces begin to dominate and a single vortex splits from the droplet. On further decreasing the field, individual vortices sequentially detach from the droplet and escape from the sample. This disintegration

\footnotetext{
${ }^{1}$ Laboratory of Condensed Matter Physics, University of Picardie, 80000 Amiens, France. ${ }^{2}$ Materials Science Division, Argonne National Laboratory, 9700 South Cass Avenue, Argonne, Illinois 60439, USA. ${ }^{3}$ Department of Physics, Stockholm University, Albanova University Center, SE-10691 Stockholm, Sweden. ${ }^{4}$ Department of Physics, University of Antwerp, Groenenborgerlaan 171, B-2020 Antwerp, Belgium. ${ }^{5}$ Department of Physics, University of Bath, Claverton Down, Bath BA2 7AY, UK. *e-mail: lukyanc@ferroix.net
} 

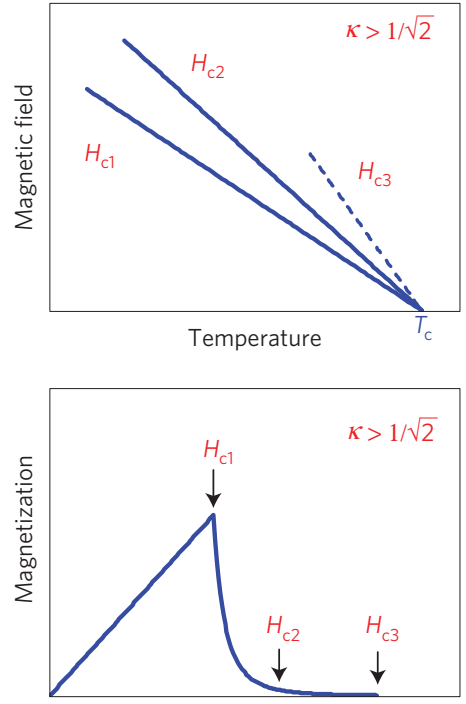

Applied magnetic field b
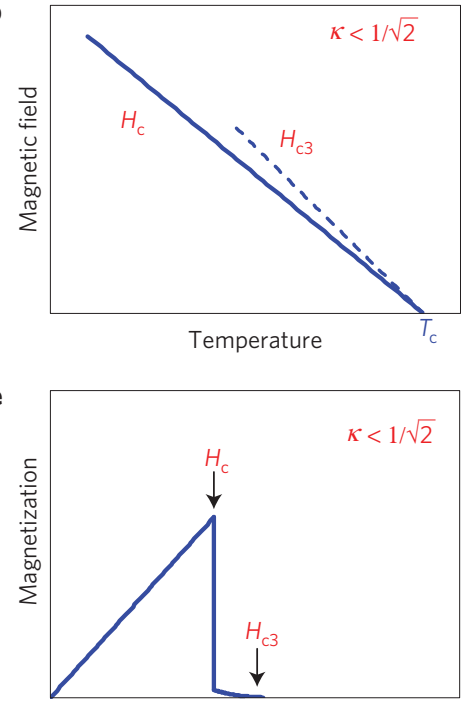

Applied magnetic field

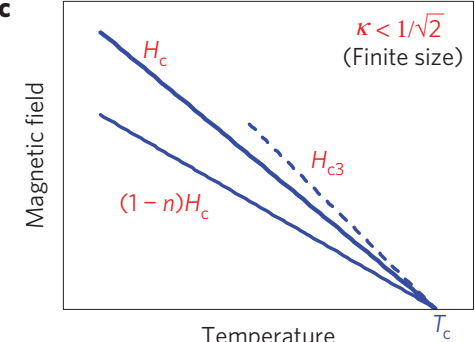

$\mathbf{f}$

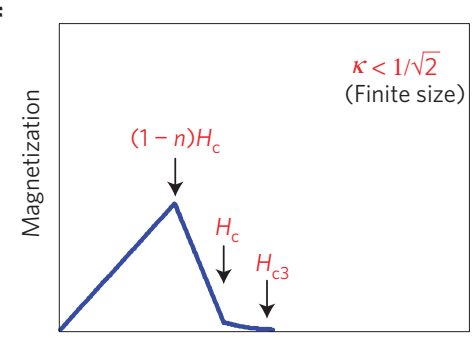

Applied magnetic field

Figure 1 | Superconducting phase diagrams and corresponding magnetization curves. a-c, Phase diagrams for type II superconductor (a), type I superconductor (b) and type I finite-size superconductor (c). d-f. The corresponding magnetization curves $M(H)$ for type II superconductor (d), type I superconductor (e) and type I finite-size superconductor (f). Note that $H_{\mathrm{c} 3}>H_{\mathrm{c}}$ holds only as long as $\kappa>0.42$.

a
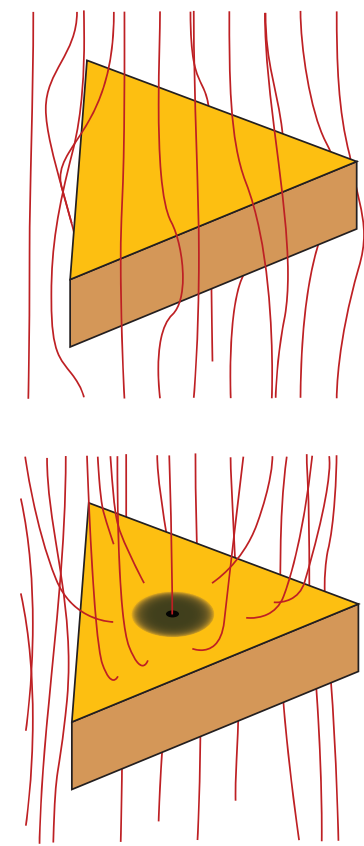

b

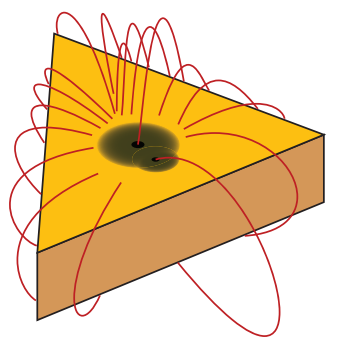

e

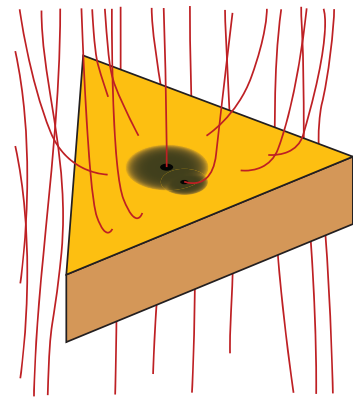

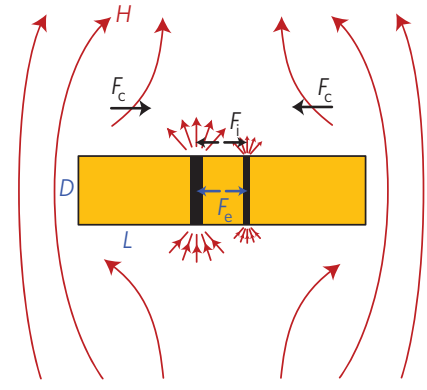

f

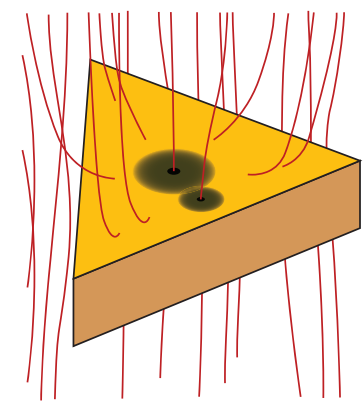

Figure 2 | Droplet fission. a-c, Distribution of the magnetic flux in the (a) Meissner state, (b) vortex droplet state showing the calculated stray fields and (c) sketch of the vortex interaction forces $\left(F_{\mathrm{e}}, F_{\mathrm{c}}\right.$, and $\left.F_{\mathrm{i}}\right)$ corresponding to the interaction energies $\left(U_{\mathrm{e}}, U_{\mathrm{c}}\right.$ and $\left.U_{\mathrm{i}}\right)$ between the droplet and a separated single vortex. d, Vortex droplet before start of the fission. e, Initial stage of separation of a single vortex from the droplet. $\mathbf{f}$, Complete separation of the vortex from the droplet.

mechanism is analogous to the instability introduced by Lord Rayleigh $^{5}$ in 1882 leading to fragmentation of charged liquid droplets due to the competition between long-range Coulomb repulsion forces and a short-range molecular attraction.

The threshold field $H_{\text {inst }}(N)$ at which the $N q$-droplet becomes unstable is determined from the instability point when the energy $U(l)$ changes its curvature and transforms from a convex function to a concave one, and equations (1)-(4) yield

$$
H_{\text {inst }}(N)=\frac{L}{\lambda_{0}}\left[\frac{\zeta(N)}{\eta} t^{1 / 2}+\gamma \frac{\alpha}{8 \eta \sqrt{N}} \frac{D}{\lambda_{0}} t\right] H_{\mathrm{c}}
$$

Direct disintegration of an $N q$-droplet into $N$ single vortices requires surmounting a higher confinement energy barrier than one-by-one vortex decoupling.

The Rayleigh instability can be observed if the field $H_{\text {inst }}(N)$ falls into the region of the existence of the vortex droplet. On the descending field branch, the vortex droplet appears as a residual of the normal state in the finite sample below the surface critical field $H_{\mathrm{c} 3}=2.39 \kappa H_{\mathrm{c}}$. Alternatively, on the ascending branch, the droplet can form as a result of the field penetration in a Meissner state. The threshold is defined by the condition that the external field at the sample edges, $H /(1-n)$, exceeds the field of first penetration 
a

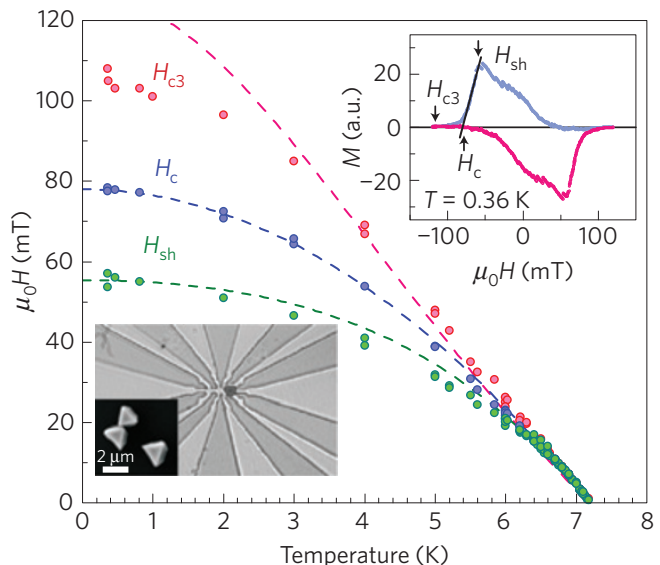

b

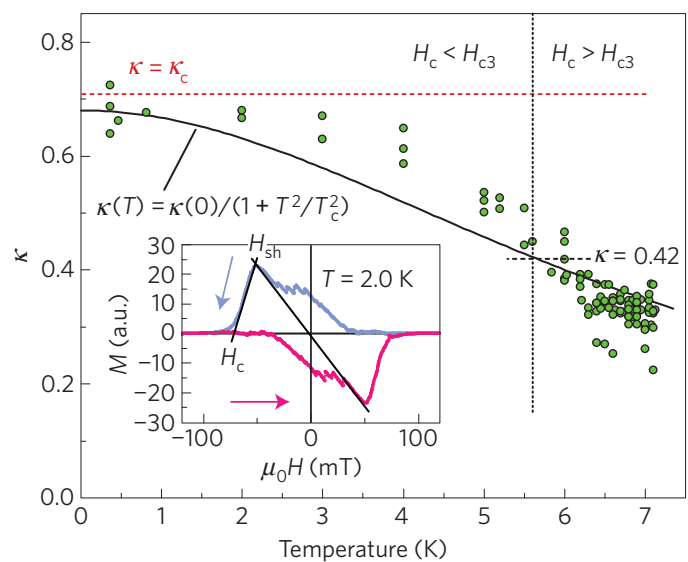

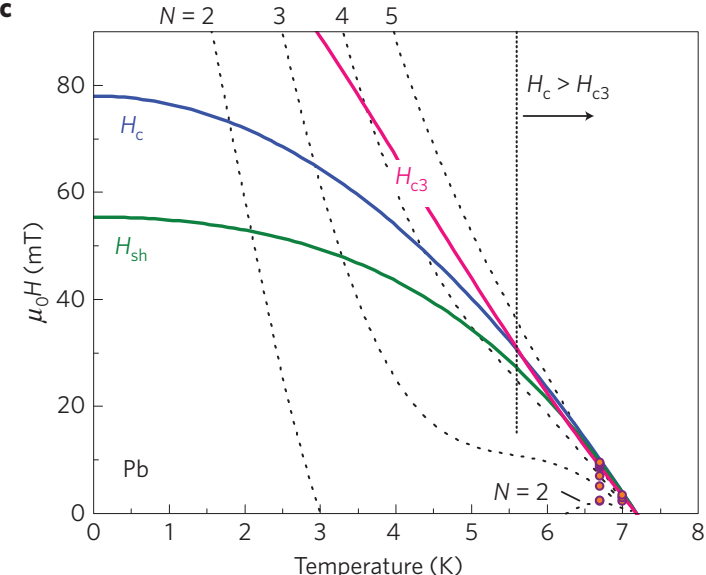

d

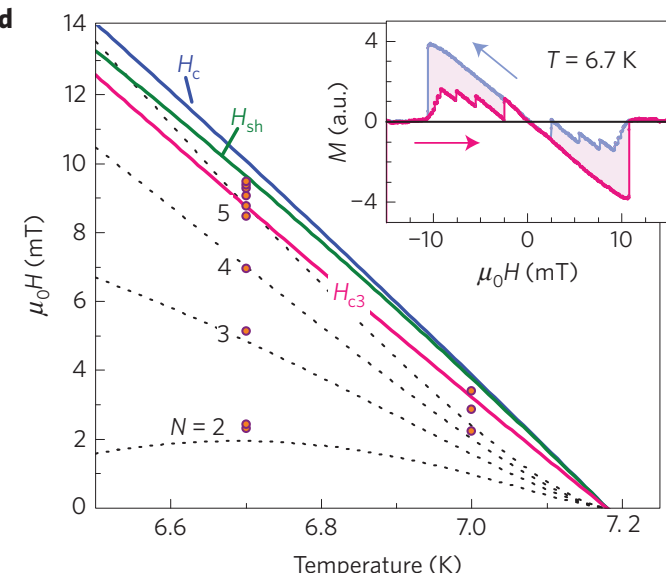

Figure 3 | Experimental phase diagrams. a, Experimental phase diagram of the meso-Pb crystal. The top inset shows the magnetization curve $M(H)$ at $T=0.36 \mathrm{~K}$ and demonstrates how $H_{\mathrm{sh}}, H_{\mathrm{c} 3}$ and $H_{\mathrm{c}}$ were obtained experimentally. The black lines are guides to the eye. Top inset: $M(H)$ at $T=0.36 \mathrm{~K}$ demonstrating how $H_{\mathrm{sh}}, H_{\mathrm{c} 3}$ and $H_{\mathrm{c}}$ were obtained experimentally. Bottom inset: experimental set-up of the crystal and the Hall probe array. b, The $\kappa(T)$ dependence of $\mathrm{Pb}$ (adopted from ref. 15). The inset shows $M(H)$, as in the top inset in $\mathbf{a}$, but at $T=2.0 \mathrm{~K}$. c, Theoretical temperature dependencies of $H_{\mathrm{c}}$, $H_{\mathrm{c} 3}$ and $H_{\text {sh }}$. Numbered dotted lines show the calculated locus of instabilities with respect to $N \rightarrow N-1$ vortex droplet fission. Red dots show the corresponding instabilities at $T=6.7 \mathrm{~K}$ and at $T=7.0 \mathrm{~K}$, associated with the data in Fig. 5 . d, Expanded interval $T_{x}<T<T_{\mathrm{c}}$. The insets in $\mathbf{b}$ and $\mathbf{d}$ show the experimental $M(H)$ dependencies in the diluted vortex gas regime at $T=2 \mathrm{~K}$ and in the metastable vortex droplet fission regime at $T=6.7 \mathrm{~K}$, respectively.

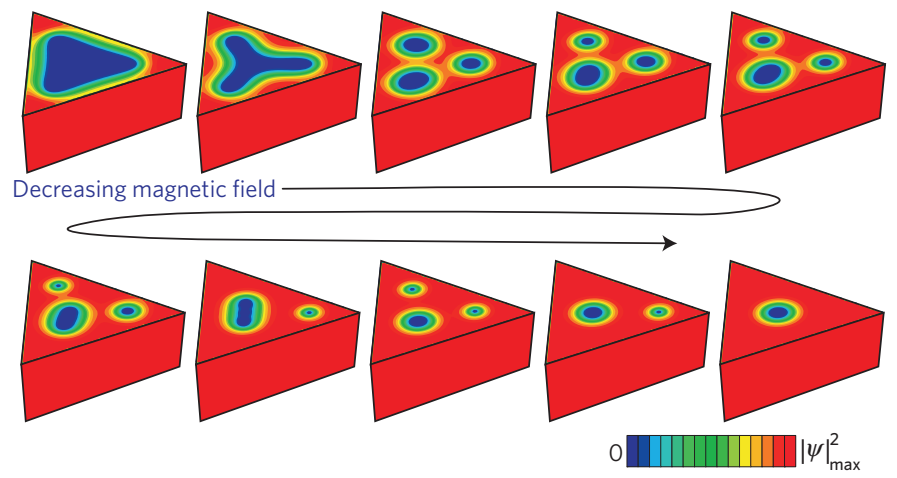

Figure 4 | Exemplary vortex configurations in the critical region. Evolution of the ground-state vortex structures on decreasing magnetic field in a mesoscale triangular superconducting Pb prism at $\kappa \sim \kappa_{\mathrm{C}}$ in the extended critical region where the vortex droplet can coexist with separate vortices. This particular simulation is performed for $T=5.5 \mathrm{~K}$, and parameters of the Pb sample $T_{\mathrm{C}}=7.2 \mathrm{~K}, \xi(0)=66 \mathrm{~nm}$ and $\lambda(0)=45 \mathrm{~nm}$, with two-fluid model temperature dependencies ${ }^{19}$.

into an infinite sample $H_{\mathrm{p}} \simeq\left(1 / 2^{1 / 4} \kappa^{1 / 2}\right)\left(1+5.44 \kappa / 1+4.78 \kappa H_{\mathrm{c}}\right)$ (ref. 14), which gives the superheating field $H_{\mathrm{sh}}=(1-n) H_{\mathrm{p}}$ for the lower bound of field penetration into a finite superconducting sample.
Criticality can be tuned by temperature variation of $\kappa(T)=\lambda(T) / \xi(T)$. In a $\mathrm{Pb}$ superconductor $\kappa(T)$ changes from $\kappa(0) \approx 0.68$ at $T=0$, which is slightly less than $\kappa_{\mathrm{c}}$, to $\kappa \simeq 0.38$ at $T=T_{\mathrm{c}}(7.2 \mathrm{~K})$ and is well described by the phenomenological 


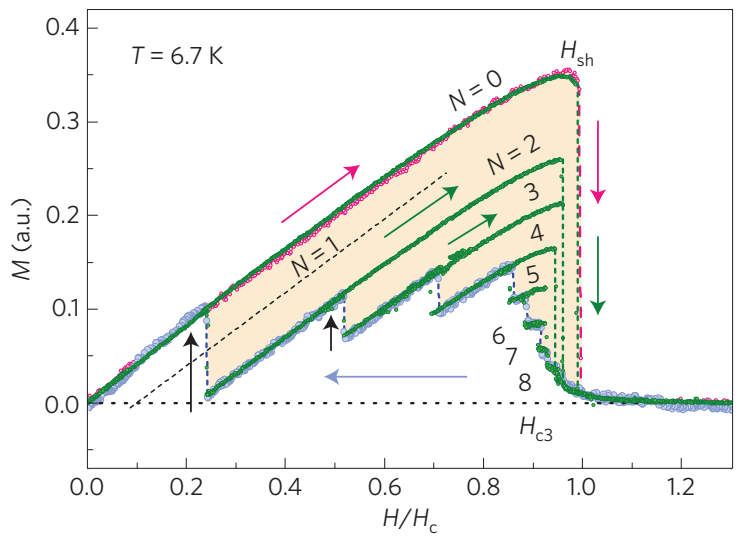

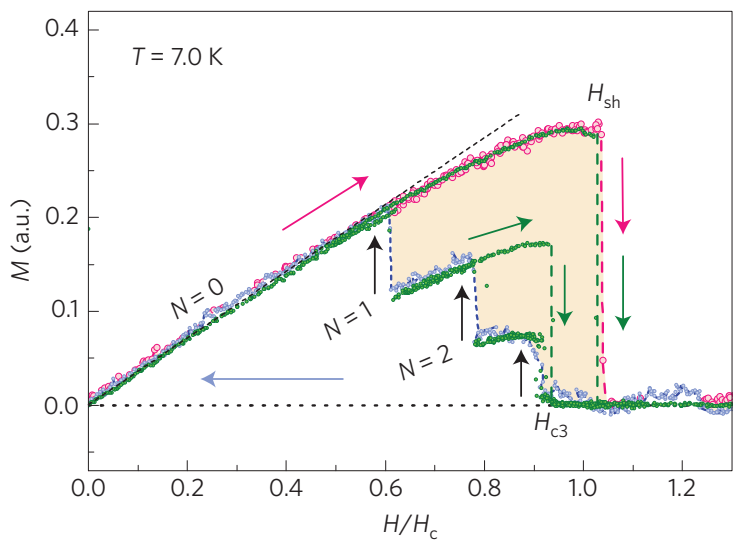

Figure 5 | Magnetization curves showing vortex droplet fission. a,b, Experimental $M(H)$ curves at $6.7 \mathrm{~K}(\mathbf{a})$ and $7.0 \mathrm{~K}(\mathbf{b})$. The applied magnetic field is normalized by $H_{\mathrm{c}}(T)=H_{\mathrm{c}}(0)\left[1-\left(T / T_{\mathrm{c}}\right)^{2}\right]$, with $H_{\mathrm{c}}(0)=78 \mathrm{mT}$ and $T_{\mathrm{c}}=7.18 \mathrm{~K}$. a.u., arbitrary units. On increasing field (red curves) the Meissner state

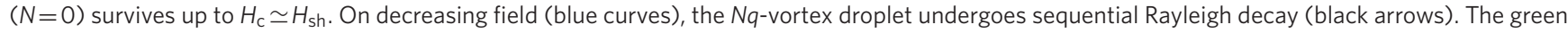
curves show the full stability ranges of the droplet states, obtained by reversing the field sweep direction.

formula $\kappa(T) \simeq \kappa(0) /\left(1+T^{2} / T_{c}^{2}\right)$ (see ref. 15 and Fig. $\left.3 \mathrm{~b}\right)$. Therefore, micrometre-sized samples of $\mathrm{Pb}$, an exemplary type I superconductor, offer a natural laboratory to study vortex droplet fission. We selected a triangular-shaped $\mathrm{Pb}$ meso-crystal with lateral side dimensions of $\sim 2.2 \mu \mathrm{m}$, thickness of $\sim 0.7 \mu \mathrm{m}$, and critical temperature $T_{\mathrm{c}}=7.18 \mathrm{~K}$ shown in the lower inset of Fig. 3a. The measurements on the crystal were done using a two-dimensional (2D) electron gas ballistic Hall micro-probe array magnetometer ${ }^{16,17}$ (see Fig. 3a lower inset). The temperature variation of $\kappa$ gives rise to the phase diagram of $\mathrm{Pb}$ shown in Fig. $3 \mathrm{c}$. The temperature dependence for $H_{\mathrm{c}}$ is standard, $H_{\mathrm{c}}(T)=H_{\mathrm{c}}(0)\left[1-\left(T / T_{\mathrm{c}}\right)^{2}\right]$ with $\mu_{0} H_{\mathrm{c}}(0) \approx 78 \mathrm{mT}$ (ref. 18). The critical fields $H_{\mathrm{sh}}$ and $H_{\mathrm{c} 3}$ are expressed through $H_{c}$ as given above with the best-fit value $n=0.37$. The curves for $H_{\mathrm{c}}, H_{\mathrm{sh}}$ and $H_{\mathrm{c} 3}$ cross pair-wise near approximately $T_{x} \simeq 6.3 \mathrm{~K}$. The dotted lines show the instability field $H_{\text {inst }}(N)$ for various $N$ calculated from equation (5). We further focus on the temperature region $T_{x}<T<T_{c}$, which is the most favourable for the experimental observation of Rayleigh instability of the vortex droplet. At $T<T_{x}$ the lines $H_{\text {inst }}(N)$ for large $N$ fall out from the range of existence of superconductivity, implying that there the droplet may become unstable with respect to splitting into single vortices. Our 3D numerical simulations, done using the phenomenologically adapted Ginzburg-Landau theory to account for the correct temperature dependence of $\kappa$ and $H_{c}$ (ref. 19), show the intermediate regime with a mixture of droplet and one-quanta vortices (see Fig. 4). Note that in the temperature range $T_{x}<T<T_{\mathrm{c}}$ where $H_{\mathrm{c} 3}<H_{\mathrm{c}}<H_{\mathrm{sh}}$, the droplet can form only in the descending field regime, because in the ascending field the sample remains in the Meissner state until the field reaches $H_{\text {sh }}$ at which superconductivity vanishes.

The temperature dependencies of $H_{c}, H_{\text {sh }}$ and $H_{c 3}$ shown in Fig. $3 c$ are in a good agreement with those of Fig. $3 a$ obtained experimentally. The data were extracted from field-dependent magnetization curves as shown in the upper inset of Fig. 3a. From $H_{\mathrm{sh}}$ and $H_{\mathrm{c}}$ one obtains the temperature dependence of the Ginzburg-Landau parameter $\kappa(T)$ through $H_{\mathrm{p}}$. The temperature dependence of the Ginzburg-Landau parameter $\kappa(T)=\lambda(T) / \xi(T)$ corresponds to the bulk behaviour (shown in Fig. 3b). Together with $H_{\mathrm{c}}(T)$ this gives a penetration $\lambda(T) \approx \lambda(0) /\left[1-\left(T / T_{\mathrm{c}}\right)^{2}\right]$ with $\lambda(0) \approx 41 \mathrm{~nm}$ and a zero temperature coherence length $\xi(0) \approx 66 \mathrm{~nm}$. To see the droplet fission, we use the individual vortex observation technique, analogous to that used in ref. 16 for observation of entrance and exit of individual vortices in small type II superconductors. The $M(H)$ dependencies at $T=2 \mathrm{~K}$ and at $T=6.7 \mathrm{~K}$ shown in the insets of Fig. $3 \mathrm{~b}$ and Fig. $3 \mathrm{~d}$ have a different character, the difference stemming from the temperature dependence of $\kappa(T)$.

At $T=2 \mathrm{~K}$, where $\kappa \simeq 0.6-0.7$ and is slightly less than $\kappa_{c}$, the lower inset of Fig. $3 \mathrm{~b}$ delineates the mixed-state-like behaviour of $M(H)$ in which the individual vortices are stabilized by the repulsion due to the stray field. First, on increasing the applied field to $H_{\mathrm{sh}} \simeq 51 \mathrm{mT}$ from the zero-field-cooled state, the absolute value of the magnetization grows proportionally to $H$ owing to Meissner screening. Beyond $H_{\mathrm{sh}}$, the magnetic flux starts to penetrate the sample and the magnetization decreases smoothly. An extrapolation of the linear drop of the absolute value of $M(H)$ to zero agrees with the bulk value $H_{\mathrm{c}} \simeq 71 \mathrm{mT}$, but the diamagnetic signature of superconductivity disappears only at $H=H_{\mathrm{c} 3} \simeq 96 \mathrm{mT}$ (Fig. 3a,b). At the reversing branch, the onset of the transition is observed at $H \approx H_{\mathrm{c} 3}$ but the magnetization remains close to zero, as long as the magnetic flux can freely leave the sample. On further change of $H$, the magnetization becomes modulated by a saw-like structure, which reflects the effect of pinning that traps vortices within the sample. The drops in $M(H)$ dependence correspond to the one-byone escape of vortices from the sample, similar to what is observed in refs 16,20 . On switching the sign of the field, vortices leave the sample, which finally falls into the Meissner state and the process repeats itself cyclically.

The full $M(H)$ curve at $T=6.7 \mathrm{~K}$, shown in the inset of Fig. 3d, is exemplary for the single droplet regime at $T_{x}<T<T_{\mathrm{c}}$ where $H_{\mathrm{c} 3}$, $H_{\mathrm{c}}<H_{\mathrm{sh}}$ are close to each other and where by tuning the field we can control the vortex droplet fission. An expanded view of one quadrant of the data is shown in Fig. 5 for $T=6.7 \mathrm{~K}$ and $7.0 \mathrm{~K}$. On the ascending field at $T=6.7 \mathrm{~K}$, the Meissner state is maintained up to $H_{\text {sh }}$. At $H=H_{\text {sh }}$ the magnetization abruptly drops to zero. Moving from high field along the descending branch, one sees that superconductivity emerges at $H_{\mathrm{c} 3}(T)$, but the system falls into a vortex droplet state.

After formation of superconductivity, $M(H)$ at the descending branch follows the envelope shape $4 \pi M=\left(n^{-1}-1\right)\left(H-H_{c}\right)$ modulated by the single quantum jumps due to one-by-one escape of vortices from the sample. Deviation from this dependence starts at $H=0.85 H_{c}$ marking the transition of the intermediate state to the metastable regime of the vortex droplet containing $N=5$ bounded vortices. On further field reduction, the disintegration of the droplet follows the scenario of instability, governed by equation (5). We marked experimentally observed values of $H_{\text {inst }}(N)$ for $N=5,4,3$ and 2 on the theoretical phase diagram in Fig. $3 \mathrm{~d}$ by filled red dots. The data show a perfect agreement with theoretical predictions. The final two-quanta jump corresponds to disappearance of the 
last 2-quanta droplet: the last $2 q$ vortex droplet splits symmetrically so that both vortices leave the sample simultaneously. A similar behaviour is observed in the $T=7.0 \mathrm{~K}$ data (Fig. 5b), where the maximum quantum number is $N=3$. Besides, near $T_{c}$ the coherence and screening lengths become comparable to the size of the sample, and the proposed theory applies only marginally. Thus, although, in general, the observations are consistent with the theoretical phase diagram of Fig. 3c,d, the experimental points appear slightly off the theoretical instability curves.

\section{Methods}

Micrometre-sized lead superconducting crystals were grown on a highly oriented pyrolytic graphite substrate synthesized using an electrochemical process, which we developed earlier ${ }^{21}$. By carefully selecting the electrodeposition parameters, we can grow a plethora of $3 \mathrm{D}$-shaped mesoscopic $\mathrm{Pb}$ superconductors with various geometries such as pyramids, pentagons, needles and brushes.

Received 26 January 2014; accepted 6 October 2014; published online 10 November 2014; corrected online 17 November 2014

\section{References}

1. Ginzburg, V. L. \& Landau, L. D. On the theory of superconductivity. Zh. Eksp. Teor. Fiz. 20, 1064-1082 (1950).

2. Abrikosov, A. A. Magnetic properties of superconductors of the second group. Zh. Eksp. Teor. Fiz. 32, 1442-1452 (1957); Sov. Phys. JETP 5, 1174-1182 (1957).

3. Bogomolnyi, E. B. The stability of classical solutions. Yad. Fiz. 24, 861-870 (1976); Sov. J. Nucl. Phys. 24, 449-454 (1976).

4. Bogomolnyi, E. B. \& Vainstein, A. I. Stability of strings in gauge Abelian theory. Yad. Fiz. 23, 1111-1114 (1976); Sov. J. Nucl. Phys. 23, 588-591 (1976).

5. Lord Rayleigh, F. R. S. On the equilibrium of liquid conducting masses charged with electricity. Phil. Mag. Ser. 5 14, 184-186 (1882).

6. Nielsen, H. B. \& Olesen, P. Vortex-line models for dual strings. Nucl. Phys. B 61, 45-61 (1973).

7. Bradlow, S. Vortices in holomorphic line bundles over closed Kahler manifolds. Commun. Math. Phys. 135, 1-17 (1990).

8. Gustafson, S., Sigal, I. M. \& Tzaneteas, T. Statics and dynamics of magnetic vortices and of Nielsen-Olesen (Nambu) strings. J. Math. Phys. 51, 015217 (2010).

9. Witten, E. From superconductors and four-manifolds to weak interactions. Bull. Amer. Math. Soc. 44, 361-391 (2007).

10. Landau, L. D. \& Lifshitz, E. M. Electrodynamics of Continuous Media (Elsevier, 1985)

11. Cren, T., Serrier-Garcia, L., Debontridder, F. \& Roditchev, D. Vortex fusion and giant vortex states in confined superconducting condensates. Phys. Rev. Lett. 107, 097202 (2011)
12. Luk'yanchuk, I. Theory of superconductors with $\kappa$ close to $1 / \sqrt{2}$. Phys. Rev. B 63, 174504 (2001).

13. Mohamed, F., Troyer, M., Blatter, G. \& Luk'yanchuk, I. Interaction of vortices in superconductors with $\kappa$ close to $1 / \sqrt{2}$. Phys. Rev. B 65, 224504 (2002).

14. Dolgert, A. J., Di Bartolo, S. J. \& Dorsey, A. T. Superheating fields of superconductors: Asymptotic analysis and numerical results. Phys. Rev. B 53, 5650-5660 (1996).

15. Cody, G. D. \& Miller, R. E. Magnetic transitions of superconducting thin films and foils. I. Lead. Phys. Rev. 173, 481-493 (1968)

16. Geim, A. K. et al. Phase transitions in individual sub-micrometre superconductors. Nature 390, 259-262 (1997).

17. Rydh, A. et al. Magnetization of a few-fluxoid lead crystal. Physica C 460, 793-794 (2007).

18. Rose-Innes, A. C. \& Rhoderick, R. H. Introduction to Superconductivity (Pergamon Press, 1969).

19. Müller, A., Milošević, M. V., Dale, S. E. C., Engbarth, M. A. \& Bending, S. J. Magnetization measurements and Ginzburg-Landau simulations of micron-size $\beta$-tin samples: Evidence for an unusual critical behavior of mesoscopic type-I superconductors. Phys. Rev. Lett. 109, 197003 (2012).

20. Geim, A. K. et al. Fine structure in magnetization of individual fluxoid states. Phys. Rev. Lett. 85, 1528-1531 (2000).

21. Xiao, Z-L. et al. Tuning the architecture of mesostructures by electrodeposition. J. Am. Chem. Soc. 126, 2316-2317 (2004).

\section{Acknowledgements}

We would like to thank N. Nekrasov for illuminating discussions. The work was supported by the US Department of Energy, Office of Science Materials Sciences and Engineering Division (V.M.V., W.K.K., U.W., R.X., M.Z., Z.L.X., G.W.C. and partially I.L. through the Materials Theory Institute), by FP7-IRSES-SIMTECH and ITN-NOTEDEV programs (I.L.), and by the Flemish Science Foundation (FWO-Vlaanderen) (M.V.M. and F.M.P.).

\section{Author contributions}

I.L., V.M.V., W.K.K. and U.W. conceived the work, I.L. and V.M.V. carried out calculations and analysed the data, M.Z. and Z.L.X. grew the samples, A.R., R.X., U.W. and W.K.K. carried out the experiment, S.J.B. provided the Hall sensor, M.V.M. and F.M.P. performed numerical simulations, I.L. and V.M.V. wrote the manuscript, A.R., U.W., M.V.M., G.W.C., S.J.B., F.M.P. and W.K.K. discussed the results and contributed to writing the manuscript.

\section{Additional information}

Supplementary information is available in the online version of the paper. Reprints and permissions information is available online at www.nature.com/reprints.

Correspondence and requests for materials should be addressed to I.L.

\section{Competing financial interests}

The authors declare no competing financial interests. 